$03,05,13$

\title{
Полупроводниковое латеральное спиновое устройство с электродами из полуметаллического ферромагнетика
}

\author{
(C) Н.А. Виглин ${ }^{1}$, В.М. Цвелиховская ${ }^{1}$, С.В. Наумов ${ }^{1}$, А.О. Шориков ${ }^{1,2}$, Т.Н. Павлов ${ }^{1}$ \\ ${ }^{1}$ Институт фризики металлов им. М.Н. Михеева УрО РАН, \\ Екатеринбург, Россия \\ ${ }^{2}$ Уральский феедеральный университет им. Б.Н. Ельцина, \\ Екатеринбург, Россия \\ E-mail: viglin@imp.uran.ru
}

Поступила в Редакцию 10 августа 2020 г.

В окончательной редакции 10 августа 2020 г.

Принята к печати 12 августа 2020 г.

В полупроводниковом спиновом устройстве с электродами, сформированными из пленки полуметаллического ферромагнетика $\mathrm{Fe}_{2} \mathrm{NbSn}$, достигнуты значения спиновой поляризации электронов, инжектированных в полупроводник $\mathrm{InSb}, P_{\mathrm{S}}=4 \%$. Это соответствует максимально возможной $P_{\mathrm{S}}$ для прозрачных контактов полупроводника и ферромагнетика, в котором поляризация $P_{\mathrm{F}}=95 \%$. Показано, что для эффективной инжекции спин-поляризованных электронов из ферромагнетика в полупроводник необходимо применять ферромагнетик, поляризация электронов в котором 100\% или формировать в интерфейсе дополнительный слой с высоким сопротивлением.

Ключевые слова: спиновая поляризация, полупроводники, спиновая инжекция, полуметаллические ферромагнетики.

DOI: $10.21883 /$ FTT.2020.12.50207.167

\section{1. Введение}

Спиновую поляризацию электронов проводимости в проводящих материалах принято обозначать $P_{\lambda}=\left(n_{\lambda \uparrow}-n_{\lambda \downarrow}\right) /\left(n_{\lambda \uparrow}+n_{\lambda \downarrow}\right)$, где индекс $\lambda$ обозначает тип материала, например, $\mathrm{F}$ - для ферромагнитного металла или $\mathrm{S}$ - для полупроводника, $n_{\lambda \uparrow}$ и $n_{\lambda \downarrow}-$ плотности электронов со спином „вверх“ и со спином „вниз“ в соответствующих материалах. Стрелкой $\uparrow$ обозначено направление оси квантования, совпадающее с направлением внешнего магнитного поля, $\downarrow-$ противоположное направление. Одним из методов создания спиновой поляризации в полупроводниках является электрическая инжекция спин-поляризованных электронов из F в S. Для успешной инжекции спин-поляризованных электронов необходимы ферромагнетики, обладающие высокой $P_{\mathrm{F}}$ собственных электронов проводимости, а инжекция через интерфейс $\mathrm{F} / \mathrm{S}$ должна быть эффективной, такой чтобы величина поляризации электронов в полупроводнике $P_{\mathrm{S}} \approx P_{\mathrm{F}}$. В ферромагнитных металлах и сплавах величина $P_{\mathrm{F}}$, не превышает нескольких десятков процентов. Например, в железе $P_{\mathrm{F}} \approx 40 \%$ [1]. В „прозрачных“, без диэлектрических прослоек между $\mathrm{F}$ и $\mathrm{S}$ контактах, эффективность инжекции существенно подавлена $\left(P_{\mathrm{S}} \ll P_{\mathrm{F}}\right)$ вследствие большой разницы величины электропроводности этих материалов. Это, так называемая проблема несовместимости проводимостей (conductivity mismatch) [2]. Эту проблему можно обойти, разместив в интерфейсе туннельный барьер или барьер Шоттки, проводимость которого много ниже проводимостей $\mathrm{S}$ и F. Например, для контакта Fe/барьер Шоттки/GaAs была получена спиновая поляризация электронов $P_{\mathrm{S}} \approx 16 \%$ [3], а в контакте $\mathrm{Co}_{0 .} \mathrm{Fe}_{0.1} / \mathrm{MgO} / \mathrm{InSb}$ удалось достичь $P_{\mathrm{S}} \approx 25 \%$, практически равную спиновой поляризации $P_{\mathrm{F}}$ в инжекторе $\mathrm{Co}_{0.9} \mathrm{Fe}_{0.1}$ [4]. Чтобы получить более высокие значения $P_{\mathrm{S}}$, электронов инжектированных в $\mathrm{S}$ из $\mathrm{F}$, необходимы ферромагнитные материалы, в которых спиновая поляризация электронов $P_{\mathrm{F}}$ выше, чем в ферромагнитных металлах. К таким материалам относятся ферромагнитные полупроводники [5], а так же полуметаллические ферромагнетики (HMF) [6]. $P_{\mathrm{F}}$ в этих материалах, в рамках ряда допущений, таких как идеальная кристаллическая структура и химический состав, температура $0 \mathrm{~K}$ и других, может быть полной или близкой к $100 \%$. В соответствии с теорией несовместимости проводимостей [2], в „прозрачных“ F/S контактах с такими материалами, спиновая инжекция весьма эффективна, но эффективность быстро падает при отклонении величины $P_{\mathrm{F}}$ от $100 \%$. В связи с этим, экспериментальное исследование реальной степени поляризации электронов в инжекторе из такого материала, является актуальной задачей. Стимулом для исследования контактной пары „F $\left(P_{\mathrm{F}} \approx 100 \%\right) / \mathrm{S}^{\text {“ }}$ так же является перспектива достижения высоких значений $P_{\mathrm{S}}$ электронов, инжектированных в полупроводник.

Полуметаллические ферромагнетики (HМF) были обнаружены при изучении электронной структуры сплавов Гейслера [6]. Большинство сплавов Гейслера относятся к группе тройных интерметаллидов со стехиометрическим составом $X Y Z$ или $X_{2} Y Z$, упорядоченных в структурах типа $\mathrm{C}_{\mathrm{b}}$ или $\mathrm{L} 2{ }_{1}$, соответственно. $X$ и $Y$ 
являются переходными металлами, а $Z$ обычно является основным элементом. $Y$ также может быть заменен редкоземельным элементом. Структура L2 1 состоит из примитивной ГЦК решетки с базой из четырех атомов. В кристаллографии L2 2 относится к типичной структуре типа $X_{2} Y Z$, обозначенной согласно номенклатуре struckturbericht, где атомы $Y$ и $Z$ занимают взаимопроникающие позиции ГЦК, а атомы $X$ занимают симметрично тетраэдрические позиции в кубической структуре. Очевидно, что комбинаций элементов, образующих сплавы Гейслера, весьма много. Часть этих возможных соединений элементов уже существует в виде сплавов, состав которых отвечает требованиям, предъявляемым к сплавам Гейслера, однако, есть комбинации атомов, формально „пригодных“, для того что бы образовать сплав Гейслера, но пока еще не существующих в виде реальных соединений. Заметим, что только некоторые из сплавов Гейслера проявляют свойства полуметаллических ферромагнетиков. Эти свойства для существующих соединений исследуются экспериментально, однако могут быть предсказаны на основе первопринципных расчетов. Характеристики существующих HMF обсуждаются в обзоре [7]. Для того чтобы в полуметаллических ферромагнетиках достигались высокие значения спиновой поляризации электронов необходимо, что бы они были стехиометрического состава и структурно упорядочены. Отсюда вытекают требования к свойствам сплава Гейслера, который предполагается применять в качестве инжектора спин-поляризованных электронов в S. Во-первых, сплав должен обладать свойствами HMF, что предварительно может быть установлено зонными расчетами. Во-вторых, рассогласование параметров кристаллических решеток сплава и $\mathrm{S}$ должно быть минимальным. Близость значений величины параметров решеток HMF и $\mathrm{S}$ значительно увеличивает вероятность „правильного“ структурного упорядочения пленки HMF при ее термообработке после осаждения. Если пленка HMF синтезируется на полупроводнике $\mathrm{InSb}$, (параметр решетки которого $a=6.47877 \AA[8]$ ), то параметр решетки HMF должен быть такой же или близкой величины, а температура отжига пленки, при которой происходит структурное упорядочение не должна превышать $400^{\circ} \mathrm{C}$ [8].

Целью настоящей работы был выбор состава НMF с указанными свойствами, синтез пленки из этого материала непосредственно на поверхности $\mathrm{InSb}$ (без промежуточного диэлектрического слоя) и исследование характеристик спиновой инжекции в спиновом устройстве с электродами из НMF.

\section{2. Методы эксперимента}

Одним из способов синтеза тонких пленок многокомпонентных соединений, таких как сплавы Гейслера, является метод магнетронного распыления мишеней, изготовленных из сплавов соответствующего состава.
Нам не удалось найти сплавы Гейслера со свойствами HMF, параметр решетки которых был бы в точности равен $a=6.48 \AA$, поэтому мы исследовали возможность приготовления мишеней из сплавов, параметр решетки которых наиболее близок к параметру а в InSb. Мишени из ферромагнитных материалов, устанавливаемые в магнетрон, представляют собой тонкие диски диаметром 50-60 mm. Толщина этих дисков не более 2-2.5 mm, поскольку диски с большей толщиной не удается намагнитить перпендикулярно плоскости так, чтобы магнетрон работал в оптимальном режиме. Сплавы Гейслера, как и большинство интерметаллидов, обладают высокой твердостью, весьма хрупкие и трудно поддаются механической обработке. Поэтому изготовление мишеней из этих материалов непростая технологическая задача. Была предпринята попытка сделать мишени из $\mathrm{Ni}_{2} \mathrm{MnAl}$ $(a=5.824 \AA)$ и $\mathrm{Ni}_{2} \mathrm{MnSn}(a=6.052 \AA)$ методом прессования порошков, изготовленных из соответствующих сплавов путем их размалывания в шаровой мельнице. Порошки смешивались с поливиниловым спиртом для связки крупинок порошка, прессовались при давлении порядка $10^{5} \mathrm{kPa}$ в цилиндрической матрице диаметром $50 \mathrm{~mm}$, затем спекались в атмосфере аргона при $T=500^{\circ} \mathrm{C}$ в течение $3 \mathrm{~h}$. Этим способом удалось получить диски диаметром $50 \mathrm{~mm}$ и толщиной $2 \mathrm{~mm}$. Однако, для применения в качестве мишеней эти диски оказались непригодны, поскольку вскоре после зажигания в магнетроне плазмы происходила интенсивная эрозия поверхности мишени и её разрушение на мелкие фрагменты.

Чтобы разрешить проблему изготовления мишени из хрупких интерметаллидов была предпринята попытка синтезировать пленки путем магнетронного распыления компонентов сплава из мозаичной мишени. Мы попытались синтезировать пленки сплавов $\mathrm{Co}_{2} \mathrm{Cr}_{0.6} \mathrm{Fe}_{0.4} \mathrm{Al}$ $(a=5.81 \AA)[9]$ и $\mathrm{Fe}_{2} \mathrm{NbSn}(a=6.08 \AA)$ [10]. Следует заметить, что значение параметра решетки $a=6.08 \AA$ для сплава $\mathrm{Fe}_{2} \mathrm{NbSn}$ было вычислено теоретически исходя из равновесного объема элементарной ячейки, так как литературных данных о величине этого параметра нет. Для этого значения $a$ в приближении GGA (приближение обобщенной градиентной поправки) была рассчитана величина поляризации $P_{\mathrm{F}}=94.324 \%$. Впоследствии после синтеза и структурирования пленки этого сплава, значение параметра решетки $a=5.78 \AA$ было вычислено на основании данных рентгеноструктурного анализа. Величина поляризации $P_{\mathrm{F}}=94.683 \% \approx 95 \%$, соответствующая скорректированному параметру $а$ даже незначительно возросла. Значения параметров $a$ для пленок $\mathrm{Co}_{2} \mathrm{Cr}_{0.6} \mathrm{Fe}_{0.4} \mathrm{Al}$ и $\mathrm{Fe}_{2} \mathrm{NbSn}$ оказались близкими по величине, но предпочтение было отдано $\mathrm{Fe}_{2} \mathrm{NbSn}$, поскольку в экспериментах по структурированию пленок кристаллическая структура в $\mathrm{Fe}_{2} \mathrm{NbSn}$ появлялась после отжига при сравнительно низкой температуре $T=200^{\circ} \mathrm{C}$. $\mathrm{B}$ пленках $\mathrm{Co}_{2} \mathrm{Cr}_{0.6} \mathrm{Fe}_{0.4} \mathrm{Al}$ кристаллическая структура возникала после отжига при $T>500^{\circ} \mathrm{C}$. При такой температуре вероятно разложение полупроводника InSb. 
Исследования величины спиновой поляризации электронов, инжектированных в полупроводник InSb из полуметаллического ферромагнетика $\mathrm{Fe}_{2} \mathrm{NbSn}$, проводились в латеральном спиновом устройстве, подобном описанному в [11], и сфабрикованном на грани (100) пластины InSb с концентрацией электронов $n=1.2 \cdot 10^{14} \mathrm{~cm}^{-3}$. Перед напылением пленки HMF на поверхность пластины InSb наносился слой фоторезиста, в котором формировалось окно размером $0.05 \times 1.8 \mathrm{~mm}$. Фоторезист задубливался при температуре $200^{\circ} \mathrm{C}$ и на него наносился второй слой фоторезиста. Методами фотолитографии во втором слое создавались окна в форме узких полос, пересекающих под прямым углом окно в первом слое фоторезиста, и с размерами, соответствующими размерам запланированных ферромагнитных контактов. После этого, не закрытые вторым слоем фоторезиста участки поверхности пластины очищались ионным травлением в атмосфере Ar. Затем методом магнетронного распыления мозаичной мишени на поверхность пластины последовательно наносились слой $\mathrm{Fe}_{2} \mathrm{NbSn}(60 \mathrm{~nm})$ и защитный слой Та $(3 \mathrm{~nm})$. На следующем этапе методом срыва удалялась пленка НMF вместе со вторым слоем фоторезиста, а оставшиеся на поверхности пластины полоски $\mathrm{Fe}_{2} \mathrm{NbSn}$ подвергались отжигу в вакууме при $T=200^{\circ} \mathrm{C}$ в течение 4 часов. Затем методом фотолитографии формировались контактные площадки и токоподводящие дорожки от этих площадок к ферромагнитным контактам. Размеры и расположение контактов в спиновом устройстве аналогичны размерам устройства, подробно описанного в работе [11]. Химический состав пленок HMF контролировался рентгеноспектральным микроанализом. Для определения состава использовались пленки, изготовленные одновременно с образцом, но напыленные на подложки из полистирола. В нашем спиновом устройстве состав пленки сплава Гейслера $\mathrm{Fe}_{2} \mathrm{NbSn}$ в атомных процентах оказался 55:23:22, то есть с небольшим отклонением от стехиометрического состава $50: 25: 25$. Для оценки эффективности спиновой инжекции производилось измерение спин-индуцированного напряжения, возникающего на детекторе при эффекте Ханле. Методика регистрации этого напряжения подробно изложена в работе [4]. Напряжение на детекторе $V(B)$ при эффекте Ханле зависит от взаимной намагниченности детектора и электронного газа вблизи детектора. Направление интегральной намагниченности электронов управляется поперечным магнитным полем, закручивающим с частотой Лармора магнитные моменты электронов. Этот метод измерения позволяет выделить разность сигналов, соответствующих параллельной намагниченности детектора и газа электронов, когда $B=0$ и тогда, когда намагниченность электронного газа направлена не параллельно намагниченности детектора и подавлена за счет расфазировки направлений магнитных моментов электронов, диффундирующих с разной скоростью в $B \neq 0$. Зависимость спин-индуцированного напряжения $V(B, d)$ от величины поперечного магнитного поля $B$ и расстояния между инжектором и детектором $d$ описывается уравнением, приведенным в работе [4]:

$$
\begin{gathered}
V(B, d)= \pm(1 / e) P_{\mathrm{F}} P_{\mathrm{S}} k_{\mathrm{B}} T \cdot \exp \left(-d \cdot \alpha / L_{S}\right) \\
\times\left[\left(\alpha \cdot \cos \left(d \cdot \beta / L_{s}\right)-\beta \cdot \sin \left(d \cdot \beta / L_{s}\right)\right) /\left(\alpha^{2}+\beta^{2}\right)\right] \\
\left.\alpha=\left[\left(1+\left(\omega_{L} \tau_{s}\right)^{2}+1\right)^{1 / 2} / 2\right]\right]^{1 / 2} \\
\beta=\left[\left(1+\left(\omega_{L} \tau_{s}\right)^{2}-1\right)^{1 / 2} / 2\right]^{1 / 2}
\end{gathered}
$$

Здесь $P_{\mathrm{S}}=P_{j} \tau_{s} \cdot J / n_{\mathrm{S}} \cdot e L_{s}-$ спиновая поляризация в $\mathrm{S}, n_{\mathrm{S}}$ - плотность электронов в $\mathrm{S} ; P_{j}=J_{s} / J-$ поляризация тока; $J=I / A-$ плотность тока, где $I-$ ток, протекающий через инжектор с площадью $A ; J_{s}$ плотность тока спинов, протекающих через инжектор; $e$ - заряд электрона; $k_{\mathrm{B}}-$ постоянная Больцмана; $\tau_{s}$ - время спиновой релаксации в $\mathrm{S} ; L_{s}$ - длина спиновой диффузии в $\mathrm{S} ; \omega_{L}=g \cdot \mu_{\mathrm{B}} \cdot B / \hbar-$ частота ларморовой прецессии спинов электронов в $\mathrm{S}, g$ есть $g$-фактор электронов проводимости в $\mathrm{S}, \mu_{\mathrm{B}}$ - магнетон Бора, $\hbar$ - постоянная Планка.

При расчете производится подгонка теоретической зависимости напряжения $V(B, d)$ к экспериментально полученной. Подгоночными параметрами являются $L_{s}$, $\tau_{s}$ и $P_{j}$. После подгонки параметров вычисляется значение $P_{\mathrm{S}}$.

\section{3. Результаты и выводы}

На рисунке изображена зависимость величины спиновой поляризации в полупроводнике $P_{\mathrm{S}}$ от плотности тока $J$ в инжекторе, вычисленная с помощью выражения (1). Кривая 1 соответствует измерениям, сделанным вскоре после изготовления устройства. Значение поляризации $P_{\mathrm{S}}$ изменялось от $1.3 \%$ до $4 \%$ при изменении плотности тока в инжекторе $J$ от 17 до $80 \mathrm{~A} / \mathrm{cm}^{2}$. На кривой 2 отображены данные полученные через несколько месяцев после изготовления устройства.

Величина $P_{\mathrm{S}}$ катастрофически снизилась и составила приблизительно $3 \cdot 10^{-4} \%$.

Оценим величину $P_{\mathrm{S}}$ с помощью выражения, полученного в работе [2] для модели прозрачных контактов между полупроводником и двумя ферромагнитными электродами.

$$
\begin{aligned}
P_{\mathrm{S}}= & P_{\mathrm{F}}\left(L_{\mathrm{F}} / x_{0}\right)\left(\rho_{\mathrm{F}} / \rho_{\mathrm{S}}\right) \\
& \times\left[2 /\left(2\left(L_{\mathrm{F}} / x_{0}\right)\left(\rho_{\mathrm{F}} / \rho_{\mathrm{S}}\right)+1\right)-P_{\mathrm{F}}\right],
\end{aligned}
$$

где $L_{\mathrm{F}}=10^{-6} \mathrm{~cm}-$ длина спиновой диффузии в ферромагнетике [2], $x_{0}=10^{-4} \mathrm{~cm}-$ толщина $\mathrm{S}, \rho_{\mathrm{F}}=370 \cdot 10^{-6} \Omega \cdot \mathrm{cm}-$ удельное сопротивление пленки $\mathrm{Fe}_{2} \mathrm{NbSn} \quad[10], \rho_{\mathrm{S}}=0.0053 \Omega \cdot \mathrm{cm}-$ удельное сопротивление $\mathrm{InSb}$ с концентрацией электронов $n=1.2 \cdot 10^{14} \mathrm{~cm}^{-3}$. Если поляризация $P_{\mathrm{F}}=95 \%$, то расчет по формуле (2) дает величину $P_{\mathrm{S}}=2.6 \%$, что близко к значениям поляризации от $1.3 \%$ до $4 \%$, полученным в спиновом латеральном устройстве сразу 


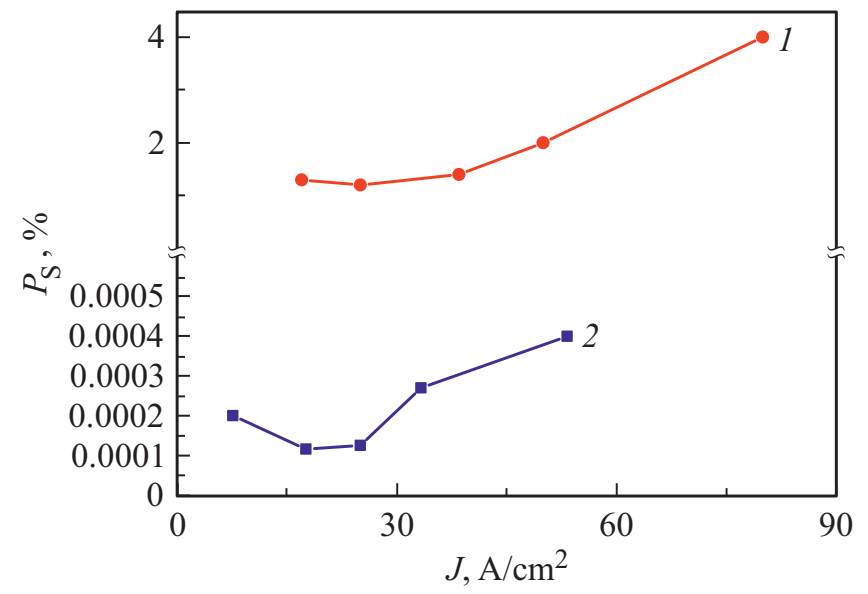

Зависимость величины спиновой поляризации в полупроводнике $P_{\mathrm{S}}$ от плотности тока $J$ в инжекторе. Расстояние между детектором и инжектором $d=10 \mathrm{mkm}$. $1-$ измерения произведены в течение недели после изготовления устройства; 2 - измерения через 8 месяцев после изготовления устройства.

после его изготовления. Можно предполагать, что в течение 1-2 недель после изготовления, пленка полуметаллического ферромагнетика $\mathrm{Fe}_{2} \mathrm{NbSn}$ с составом, близким к стехиометрическому, синтезированная на грани (100) полупроводника InSb, имеет структуру $\mathrm{L} 21$ и максимальный $P_{\mathrm{F}}=95 \%$. Отметим, что величина $P_{\mathrm{S}}$, вычисленная с помощью выражения (2), снижается до сотых долей процента при уменьшении $P_{\mathrm{F}}$. Мы оценили в приближении GGA значение поляризации $P_{\mathrm{F}}$ для разных случаев отклонения от стехиометрии в сплаве $\mathrm{Fe}_{2} \mathrm{NbSn}$. Например, когда половина атомов $\mathrm{Sn}$ заменяются вакансиями, ячейка при расчете имеет формулу $\mathrm{Fe}_{4} \mathrm{Nb}_{2} \mathrm{Sn}+$ vac и $P_{\mathrm{F}}=0.055 \%$. Расчет по формуле (2) дает значения $P_{\mathrm{S}}=7.6 \cdot 10^{-5 \%}$. Если места вакансий займут атомы железа, то для ячейки $\mathrm{Fe} 5 \mathrm{Nb} 2 \mathrm{Sn}$ величина $P_{\mathrm{F}}=24 \%$ и рассчитанное по формуле (2) значение $P_{\mathrm{S}}=4.4 \cdot 10^{-2 \%}$. Изменение характеристик пленок со временем, которое мы наблюдали, может быть вызвано структурными изменениями, связанными с низкотемпературной диффузией атомов в интерфейсе стимулированной, например, большим рассогласованием параметров решеток $(\sim 6 \%)$. Так же известно, что структурный и химический беспорядок очень зависят от условий напыления и отжига пленок [12].

Таким образом, осуществлена первая реализация спинового устройства с HMF-электродами на полупроводнике InSb. Сравнение значений экспериментально измеренной спиновой поляризации $P_{\mathrm{S}}=1.3-4 \%$, электронов инжектированных в полупроводник, и $P_{\mathrm{S}}=2.6 \%$, рассчитанной теоретически для прозрачного контакта полупроводника и ферромагнетика с $P_{\mathrm{F}}=95 \%$, позволяет утверждать, что пленка $\mathrm{HMF} \mathrm{Fe}_{2} \mathrm{NbSn}$ сразу после синтеза и структурирования упорядочена в структуру L2 $2_{1}$, так как только в сплавах стехиометрического состава и совершенной кристаллической структурой L21 может быть высокое значение $P_{\mathrm{F}}$ (Для $\mathrm{Fe}_{2} \mathrm{NbSn}$ поляризация $\left.P_{\mathrm{F}}=95 \%\right)$. Химический состав и характеристики электронной системы соединения $\mathrm{Fe}_{2} \mathrm{NbSn}$ были предсказаны теоретически, пока сплав существует в виде тонких пленок.

Стабилизация характеристик пленок HMF от времени зависит от условий их приготовления и предполагает дополнительные исследования. Экспериментально показано, что спиновая инжекция в прозрачном контакте из ферромагнетика с очень высокой $\left(P_{\mathrm{F}}=95 \%\right)$, но все же не $100 \%$ поляризацией электронов, не эффективна. Для получения эффективной спиновой инжекции в полупроводниковом латеральном спиновом устройстве с прозрачными контактами необходимо, чтобы электроды обладали $P_{\mathrm{F}}=100 \%$. Следует сказать, что в случае применения ферромагнетиков с высокой, но не полной поляризацией электронов по спину, например $\mathrm{HMF}$, поляризация электронов в которых $P_{\mathrm{F}}<100 \%$, эффективную инжекцию можно получить, если использовать дополнительные прослойки, например, туннельный барьер или барьер Шоттки, как это было продемонстрировано при инжекции спин-поляризованных электронов из $\mathrm{Co}_{2} \mathrm{MnGa}$ через барьер Шоттки в GaAs [13].

\section{Финансирование работы}

Работа выполнена в рамках государственного задания Минобрнауки России (тема „Спин“, № ААААА18-118020290104-2) при частичной поддержке РФФИ (проект № 19-02-00038).

\section{Конфликт интересов}

А вторы заявляют, что у них нет конфликта интересов

\section{Список литературы}

[1] R.J. Soulen Jr., J.M. Byers, M.S. Osofsky, B. Nadgorny, T. Ambrose, S.F. Cheng, P.R. Broussard, C.T. Tanaka, J. Nowak, J.S. Moodera, A. Barry, J.M.D. Coey. Science 282, 85 (1998).

[2] G. Schmidt, D. Ferrand, L.W. Molenkamp, A.T. Filip, B.J. van Wees. Phys. Rev. B 62, R4790(R) (2000).

[3] X. Lou, C. Adelmann, S.A. Crooker, E.S. Garlid, J. Zhang, S.M. Reddy, S.D. Flexner, C.J. Palmstr $\varnothing \mathrm{m}$, P.A. Crowell. Nature Phys. 3, 197 (2007).

[4] Н.А. Виглин, В.М. Цвелиховская, Н.А. Кулеш, Т.Н. Павлов, Письма в ЖЭТФ, 110248 (2019). [N.A. Viglin, V.M. Tsvelikhovskaya, N.A. Kulesh, T.N. Pavlov. JETP Lett. 110273 (2019).]

[5] Э.Л. Нагаев. Физика магнитных полупроводников. Наука, M.(1979).

[6] R.A. de Groot, F.M. Mueller, P.G. van Engen, K.H.J. Buschow. Phys. Rev. Lett. 502024 (1983).

[7] M.I. Katsnelson, V.Yu. Irkhin, L. Chioncel, A.I. Lichtenstein, R.A. deGroot, Rev. Mod. Phys. 801 (2008). 
[8] А.Я. Нашельский. Техгология спецматериалов электронной техники. Металлургия, М. (1993), 368 с. [A.Ya. Nashel'sky. Technology of Special Materials of Electron Technique Metallurgiya, M. (1993). p. 368.].

[9] A.D. Rata, H. Braak, D.E. Bürgler, S. Cramm, C.M. Schneider. Eur. Phys. J. 52445 (2006).

[10] Н.А. Виглин, В.В. Марченков, М.А. Миляев, С.В. Наумов, Е.И. Патраков, А.О. Шориков, Е.И. Шредер, К.А. Белозерова. ФММ 1141 (2013). [N.A. Viglin, V.V. Marchenkov, M.A. Milyaev, S.V. Naumov, E.I. Patrakov, A.O. Shorikov, E.I. Shreder, K.A. Belozerov. Phys. Met. Metallography 114 1003 (2013).

[11] N.A. Viglin, V.V. Ustinov, S.O. Demokritov, A.O. Shorikov, N.G. Bebenin, V.M. Tsvelikhovskaya, T.N. Pavlov, E.I. Patrakov. Phys. Rev B 96, 235303 (2017).

[12] Y.V. Kudryavtsev, V.A. Oksenenko, Y.P. Lee, Y.H. Hyun, J.B. Kim, J.S. Park, S.Y. Park, J. Dubowik. Phys. Rev. B 76, 024430 (2007).

[13] M.C. Hickey, C.D. Damsgaard, S.N. Holmes, I. Farrer, G.A.C. Jones, D.A. Ritchie, C.S. Jacobsen, J.B. Hansen, M. Pepper. Appl. Phys. Lett. 92, 232101 (2008).

Редактор К.В. Емцев 